\title{
Phage matrix for isolation of glioma cell membrane proteins
}

\author{
Tatiana I. Samoylova ${ }^{1}$, Nancy R. Cox ${ }^{1}$, Nancy E. Morrison ${ }^{1}$, Ludmila P. Globa ${ }^{1}$, \\ Victor Romanov ${ }^{2}$, Henry J. Baker ${ }^{1}$, and Valery A. Petrenko ${ }^{1}$
}

BioTechniques 37:254-260 (August 2004)

\begin{abstract}
Cell-binding ligands for RG2 rat glioma were identified in our recent study from a library of peptides that are displayed as fusion molecules on phage particles. Here, one of the phage clones was used to affinity purify those cell membrane components to which the displayed peptides bind. This phage clone, displaying the ELRGDSLP peptide, was shown to recognize glioma cells specifically in comparison to control phage-expressing peptides of either similar or irrelevant sequences. Blocking experiments with synthetic RGDS peptide demonstrated that the phage-glioma cell recognition occurs via the RGD motif known to be present in many integrin-binding proteins. To form an affinity matrix that would bind to glioma cell membrane molecules, ELRGDSLP phage particles were cross-linked using dextran polymer. Whole cell lysate from RG2 rat glioma cells was passed through the matrix, resulting in the isolation of cell membrane components having strong affinity to the peptides on phage and molecules associated with those components. One of the isolated proteins was found to be CD44s, a cell surface adhesion molecule involved in glioma cell invasion and migration, which likely formed a complex with an RGD-binding integrin. Cell membrane proteins isolated with this innovative approach could be used for the design of cell-specific anticancer treatments.
\end{abstract}

\section{INTRODUCTION}

Proteins expressed on the surfaces of cell membranes are of great interest as targets for cell-specific antitumor therapies and may be useful in immunotherapy protocols. The isolation of such proteins is usually achieved by immunoprecipitation or affinity purification on sorbents with immobilized antibody or peptide ligands. The affinity ligands used for the isolation may be recruited from phage display libraries expressing antibody fragments (antibody phage display libraries) or random peptides (random peptide phage display libraries) that are specific for those proteins (1-3). Unlike other techniques, phage display offers the opportunity to rapidly identify cell-specific ligands to multiple as well as unknown cell surface targets (4). This feature is especially important in ligand development for anticancer therapies because cancers may comprise heterogeneic cells that express different cellsurface markers. Malignant gliomas are fatal brain tumors composed of heterogeneic cells expressing molecular markers that differ not only from patient to patient but also from cell to cell within the tumor mass. Cell heterogeneity and a limited number of truly glioma-specific receptors identified thus far $(5,6)$ are among those factors that prevent the development of targeting cytotoxic agents that are effective in the majority of patients (7).

The purpose of this study was to develop a rapid and inexpensive method for the isolation of targeted glioma cell membrane proteins using a rodent glioma cell line as a model. A landscape phage display peptide library (8) was applied in our previous study (9) to select cell-specific phage carrying gliomatargeting ligands. In this library, each phage particle expresses thousands of copies of a foreign peptide as a part of phage major coat protein $\mathrm{pVIII}$. Because the body of the phage is the carrier of multiple copies of the ligand (peptide) that recognize the cell surface components, cross-linking multiple identical phage creates an affinity matrix for the isolation of the bound molecules. This technique was described by Smith et al. (10) to effectively affinity purify monospecific antibody to the S54 epitope on bacteriophage $\mathrm{T} 4$ from the serum of a mouse immunized with whole $\mathrm{T} 4$ virions. Here we describe the use of crosslinked phage to isolate phage-binding surface molecules from whole cell lysates of the target cell, RG2 rat glioma, for proof of concept. Because phage can be easily propagated in bacterial cultures, the use of phage carrying cellbinding peptides for protein isolation is more cost-effective than the production of synthetic peptides or antibodies. In addition, the protocol does not require modification of the phage (e.g., biotinylation) that could minimize the presence of nonspecifically isolated proteins.

\section{MATERIALS AND METHODS}

\section{Cells}

The cell lines used in this study included two rat gliomas, RG2 (CRL2433) and F98 (CRL-2397), rat hepatocytes (CRL-1442), and rat astrocytes (CRL-2006). All were purchased from the ATCC (Manassas, VA, USA) and cultured as recommended by the vendor.

\footnotetext{
${ }^{1}$ Auburn University, Auburn, AL and ${ }^{2}$ SUNY at Stony Brook, Stony Brook, NY, USA
} 


\section{Phage Clones}

The ELRGDSLP (hereafter referred to as Gli27) phage clone used for preparation of the phage affinity matrix was selected from a landscape f8-1/8mer phage display library because of its binding to rat RG2 glioma cells (9). This library is composed of $2 \times 10^{9}$ variants of fd-tet phage, which express foreign peptides as part of the major coat protein pVIII (8). Four phage clones were used as controls in binding assays for phage specificity. One control was a wild-type phage vector (f8-5) that does not express any foreign peptides. Two phage clones, $1 \mathrm{~F} 20$ and $1 \mathrm{~F} 29$, were isolated in a previous study from the same library for two different binding sites of fibrinogen (11). 1F20 phage displays the EAGPRSAP peptide, and 1F29 displays the EGYLRPDT peptide. The fourth control phage, 1G40 with the DTFAKSMQ peptide, was selected from the same library against $\beta$-galactosidase (12). All general methods of handling fd-tet phage, including propagation, purification, titering, production of pure phage clone, and isolation of phage DNA were as previously described in detail in Phage Display: A Laboratory Manual (13).

\section{Phage-Binding Assay}

RG2-selected phage clone Gli27 and the control phage clones f8-5, $1 \mathrm{~F} 20,1 \mathrm{~F} 29$, and $1 \mathrm{G} 40$ were assayed for binding to RG2 glioma cells. Briefly, RG2 cells were grown in 25$\mathrm{cm}^{2}$ flasks for approximately $48 \mathrm{~h}$ to subconfluence. One hour prior to the application of phage, standard medium was replaced with serum-free medium. Each phage clone $\left[10^{9}\right.$ colony-forming unit (cfu)/flask] was added to the cells in $2 \mathrm{~mL}$ of washing/blocking buffer $[0.1 \%$ bovine serum albumin (BSA), $0.1 \%$ Tween $^{\circledR} 20$ in Dulbecco's modified Eagle's medium (DMEM)], and incubated for $1 \mathrm{~h}$ at room temperature. The media with unbound phage were removed carefully from the flasks, and cells were washed eight times with 4 $\mathrm{mL}$ of cold washing/blocking buffer for $5 \mathrm{~min} / \mathrm{wash}$. To recover cell-associated phage, the cells were lysed with $1 \mathrm{~mL}$ lysis buffer [2\% deoxycholic acid (sodium salt), $10 \mathrm{mM}$ Tris- $\mathrm{HCl}$,
pH 8.0, 2 mM EDTA (14)] for $10 \mathrm{~min}$ at room temperature. The relative binding of the phage to the cells was determined by phage titering using the K91BluKan strain of Escherichia coli as host bacteria. All analyses were done in triplicate.

\section{Blocking with RGDS Synthetic Peptide}

RG2 cell cultures were grown in 25 $\mathrm{cm}^{2}$ flasks to form subconfluent monolayers. Two milliliters of RGDS peptide (Sigma, St. Louis, MO, USA) in DMEM at concentrations $0.058,0.58,1.16$, or $2.31 \mathrm{mM}$ were added to the cells and incubated for $1 \mathrm{~h}$ at $4^{\circ} \mathrm{C}$. After that, Gli27 phage $\left(10^{9} \mathrm{cfu}\right.$ in $1 \mathrm{~mL}$ washing/blocking buffer) was added to the cells for 1 $\mathrm{h}$ at room temperature. Unbound phage was removed, and the cells were washed six times for 2 min each with cold washing/blocking buffer. The phage associated with the cells was recovered with the lysis buffer as described above and quantified via titering.

\section{Propagation of Phage}

Gli27 phage was propagated as described by Bonnycastle et al. (15). Briefly, $1 \mathrm{~L}$ of NZY (1\% N-Z-amine A, $0.5 \%$ yeast extract, $0.5 \% \mathrm{NaCl}, \mathrm{pH}$ 7.5) containing $20 \mu \mathrm{g} / \mathrm{mL}$ tetracycline was inoculated with a single colony of cells harboring Gli27 phage. The culture was grown for approximately $24 \mathrm{~h}$ to reach stationary phase. The culture was then centrifuged at $4200 \times g$ for 10 min at $4^{\circ} \mathrm{C}$, and supernatant containing phage was collected and recentrifuged at $11,300 \times g$ for $10 \mathrm{~min}$ at $4^{\circ} \mathrm{C}$. Cleared supernatant was transferred into a fresh centrifuge bottle, and a 0.15 volume of polyethylene glycol (PEG)/ $\mathrm{NaCl}$ (16.7\% PEG, 3.3 M NaCl) was added, mixed thoroughly by inversions, and left overnight at $4^{\circ} \mathrm{C}$. Phage was pelleted by centrifugation $(11,300 \times g$ for $40 \mathrm{~min}$ at $4^{\circ} \mathrm{C}$ ), and the pellet was resuspended in $10 \mathrm{~mL}$ of phosphatebuffered saline (PBS). The phage suspension was clarified by centrifugation $\left(12,000 \times g\right.$ for $10 \mathrm{~min}$ at $\left.4^{\circ} \mathrm{C}\right)$, and cleared supernatant was transferred to a fresh centrifuge tube for the second phage precipitation with $\mathrm{PEG} / \mathrm{NaCl}$. Phage collected by centrifugation as 
described above was resuspended in 2 $\mathrm{mL}$ of coupling buffer $(65 \mathrm{mM} \mathrm{NaCl}$, $130 \mathrm{mM} \mathrm{NaH} \mathrm{PO}_{4}, \mathrm{pH}$ 6.9) for the preparation of cross-linked phage. Phage concentration was measured by spectrophotometry and calculated in virions per milliliter, assuming that 1 o.u. $_{269}=6.7 \times 10^{12}$ virions $/ \mathrm{mL}(13)$.

\section{Preparation of Cross-Linked Phage}

Cross-linked phage was prepared by a method that has been previously described (10). Briefly, one milliliter of phage $\left(2 \times 10^{14}\right.$ virions $\left./ \mathrm{mL}\right)$ in coupling buffer was mixed with $10 \mu \mathrm{L}$ of N-hydroxysuccinimide-dextran (NHSdextran; a water-soluble multifunctional dextran polymer custom-synthesized by CarboMer, Westborough, MA, USA) in dimethylsulfoxide. The reaction mixture was vortex mixed immediately, $150 \mu \mathrm{L} 50 \%$ PEG in water were added, and the mixture was vortex mixed again. The reaction mixture was rotated overnight at room temperature. Unreacted N-hydroxysuccinimide (NHS) groups were quenched by the addition of $8 \mathrm{~mL}$ of $1 \mathrm{M}$ Tris- $\mathrm{HCl}, \mathrm{pH} 9.5$, and $890 \mu \mathrm{L} 5$ $\mathrm{M} \mathrm{NaCl}$. Rotation was continued for an additional $1-2 \mathrm{~h}$ at room temperature. Cross-linked phage was pelleted by centrifugation $(12,000 \times g)$ for 15 $\mathrm{min}$. The pellet was resuspended in 20 $\mathrm{mL}$ of Tris-buffered saline (TBS) and washed 5-6 times by centrifugation as described above, decanting the supernatant and resuspending the pellet in fresh TBS. The final pellet of crosslinked phage was suspended in $1 \mathrm{~mL}$ TBS and stored at $4{ }^{\circ} \mathrm{C}$. The concentration of the cross-linked phage was determined spectrophotometrically, as previously described (10).

\section{Isolation of Cell Membrane Proteins}

RG2 cells were grown in six $75 \mathrm{~cm}^{2}$ flasks to obtain confluent monolayers. The medium was removed, and the cells were rinsed with ice-cold PBS buffer. The cells were lysed in $5 \mathrm{~mL} / \mathrm{flask}$ Tris$\mathrm{HCl}$ buffer $(10 \mathrm{mM}$, pH 7.5) containing $140 \mathrm{mM} \mathrm{NaCl}, 10 \mathrm{mM}$ octylglucoside (Sigma), and $170 \mu \mathrm{L}$ protease inhibitor cocktail (Sigma) for $15 \mathrm{~min}$ at $4^{\circ} \mathrm{C}$. The lysates were combined and clarified by centrifugation at $23,400 \times g$ at $4^{\circ} \mathrm{C}$ for 10 min, and the supernatant was used for the isolation of glioma cell membrane proteins. In some experiments, intact RG2 cells were biotinylated with the EZ-Link Sulfo-NHS-LC-LC Biotin (Pierce, Rockford, IL, USA) prior to lysis.

Cross-linked Gli27 (10 14 virions) prepared as described above was incubated with the lysate of glioma cells overnight at $4{ }^{\circ} \mathrm{C}$ with rotation. Complexes of cross-linked phage with cell components were pelleted at 20,000x $g$ for $15 \mathrm{~min}$ at $4^{\circ} \mathrm{C}$. The pellet was washed twice by resuspension in 30 $\mathrm{mL}$ of lysis buffer and recentrifuged as above. Cell components bound to the phage were eluted with $200 \mu \mathrm{L}$ of elution buffer $(0.1 \%$ BSA, $0.2 \mathrm{M}$ glycine$\mathrm{HCl}, \mathrm{pH} 2.2$ ) for $15 \mathrm{~min}$ at $4^{\circ} \mathrm{C}$. Phage matrix was separated from the eluate by centrifugation for $10 \mathrm{~min}$ at maximum speed $(16,000 \times g)$ in a microcentrifuge. The eluate was transferred to a fresh tube and neutralized by the addition of $37.5 \mu \mathrm{L}$ of $1 \mathrm{M}$ Tris- $\mathrm{HCl}, \mathrm{pH} 9.0$.

\section{Western Blot Analysis}

To detect proteins from biotinylated RG2 cells, cell lysate, clarified cell lysate, and proteins isolated via Gli27 phage matrix were separated by $7.5 \%$ sodium dodecyl sulfate polyacrylamide gel electrophoresis (SDS-PAGE), blotted onto polyvinylidene difluoride (PVDF) membranes (Immobilon ${ }^{\text {TM }}$-P PVDF; Millipore, Bedford, MA, USA), probed with horseradish peroxidase (HRP)-labeled streptavidin, and visualized with enhanced chemiluminescence (ECL Plus ${ }^{\mathrm{TM}}$ Western Blotting Detection Reagents; Amersham Biosciences, Piscataway, NJ, USA).

To identify Gli27 phage-isolated proteins, an aliquot of the nonbiotinylated protein preparation obtained using the protocol above and whole cell lysates (controls) were separated by SDSPAGE, blotted onto PVDF membranes, and probed with antibodies $\alpha v, \beta 3$, and $\beta 1$ integrins and for hyaluronic acid receptor (CD44). To probe for integrin $\alpha v$, anti-integrin $\alpha v(Q-20)$ antibodies (Santa Cruz Biotechnology, Santa Cruz, CA, USA) at a 1:500 dilution were used, followed by biotin-conjugated rabbit anti-goat IgG $(\mathrm{H}+\mathrm{L}$; Southern Biotechnology Associates, Birmingham, AL, USA) at a 1:10,000 dilution, and then with streptavidin-HRP at a 1:20,000 dilution (Amersham Biosciences). Antiintegrin $\beta 3$ antibodies (C-20; Santa Cruz Biotechnology) were applied at a 1:100 dilution, and HRP-conjugated rabbit $\mathrm{F}\left(\mathrm{ab}^{\prime}\right) 2$ anti-goat $\operatorname{IgG}(\mathrm{H}+\mathrm{L})$ at a dilution of 1:8000 as secondary antibodies. AntiCD29 (integrin $\beta 1$ chain) antibodies (BD Biosciences Pharmingen, Franklin Lakes, NJ, USA) were used to probe the blots at a dilution of 1:100, followed by HRP-conjugated goat anti-armenian hamster IgG $(\mathrm{H}+\mathrm{L}$; Jackson ImmunoResearch Laboratories, West Grove, PA, USA) at a dilution of 1:10,000. To detect CD44 protein, the blots were incubated with either anti-HCAM (N-18) antibodies (Santa Cruz Biotechnology) at a 1:100 dilution or anti-CD44H (Pgp$1 \mathrm{H}-\mathrm{CAM}$ ) antibodies (BD Biosciences Pharmingen) at a 1:100 dilution. Following this step, the blots were incubated with HRP-conjugated rabbit $\mathrm{F}\left(\mathrm{ab}^{\prime}\right) 2$ anti-goat IgG at a 1:5000 dilution. In all cases, the HRP reactions were visualized using the ECL Plus Western Blotting Detection Reagents.

\section{RESULTS}

\section{Gli27 Phage Clone}

Gli27 phage clone with the ELRGDSLP motif was selected in our recent study (9) for RG2 rat glioma cells from a landscape f8-1/8-mer phage display library composed of $2 \times 10^{9}$ different phage clones. Each clone in this library has a random 22 nucleotide insert in gene VIII and, therefore, expresses a foreign 8-mer peptide as a part of the major coat protein pVIII. To select for glioma, an aliquot of this library in blocking buffer was incubated with RG2 rat glioma cells. During the process of incubation, phage clones carrying ligands for glioma cell membrane markers became bound to the cells, while nonspecific clones remained in the buffer and were removed via washing steps. Phage bound to the cells was collected, amplified in bacteria, and applied to the cells again to achieve maximum enrichment in cell-binding clones. Following four rounds of phage selection, Gli27 phage was isolated from the whole cell lysate, propagated as an individual clone, and tested for glioma binding.

To ensure that Gli27 showed phage 
specificity for RG2 glioma cells, the clone was assayed for binding to the cells in comparison with four controls (Figure 1). The controls were the wildtype vector $\mathrm{f} 8-5$ that does not display any foreign peptide, and three phage clones $(1 \mathrm{G} 40,1 \mathrm{~F} 20$, and $1 \mathrm{~F} 29)$ that were selected from the same library in unrelated experiments. These control phage clones were chosen for the study because they express peptides with a similar (EAGPRSAP and EGYLRPDT) or irrelevant (DTFAKSMQ) to ELRGDSLP sequence. As seen in the Figure 1, ELRGDSLP-carrying phage bound with high affinity to RG2 glioma cells, while the relative numbers of cell-bound phage for all controls appeared to be negligible. Based on these results, we concluded that Gli27 phage presenting ELRGDSLP peptide could be used to create a matrix for the affinity purification of glioma cell membrane proteins.

\section{Competitive Binding Studies}

Glioma-binding phage clone Gli27 displays the peptide ELRGDSLP with the RGD motif as a part of the sequence. RGD is a binding sequence in many extracellular matrix proteins that are known to interact with glioma cells through cell membrane receptor integ-

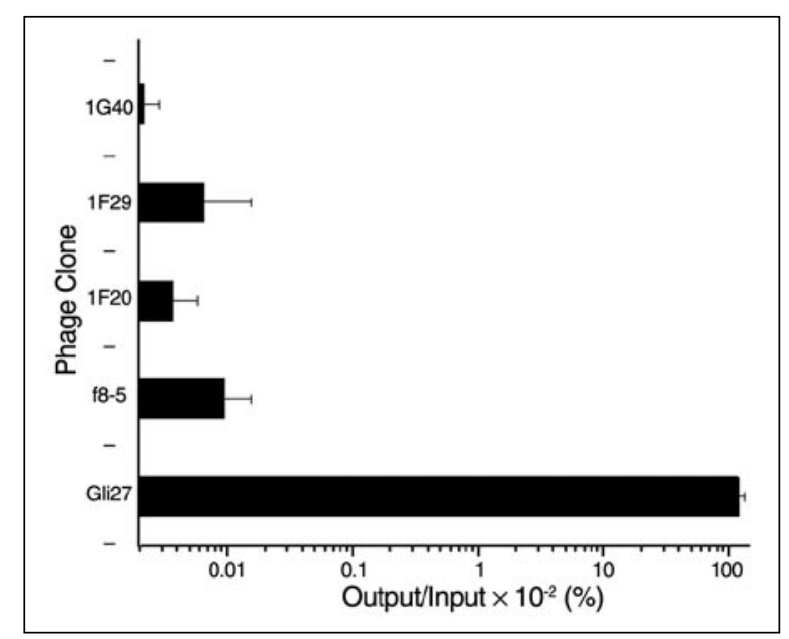

Figure 1. Binding of Gli27 phage clone to RG2 glioma cells. Gli27 and control phage clones (1G40,1F29, 1F20, and f8-5) were recovered by lysis after incubation with RG2 cells. Phage titers in the lysates were determined by the infection of bacteria and plotted as output to input ratios (x-axis) against different phage clones (y-axis). Data represent the means of bound phage from triplicate analyses $( \pm \mathrm{sD})$.

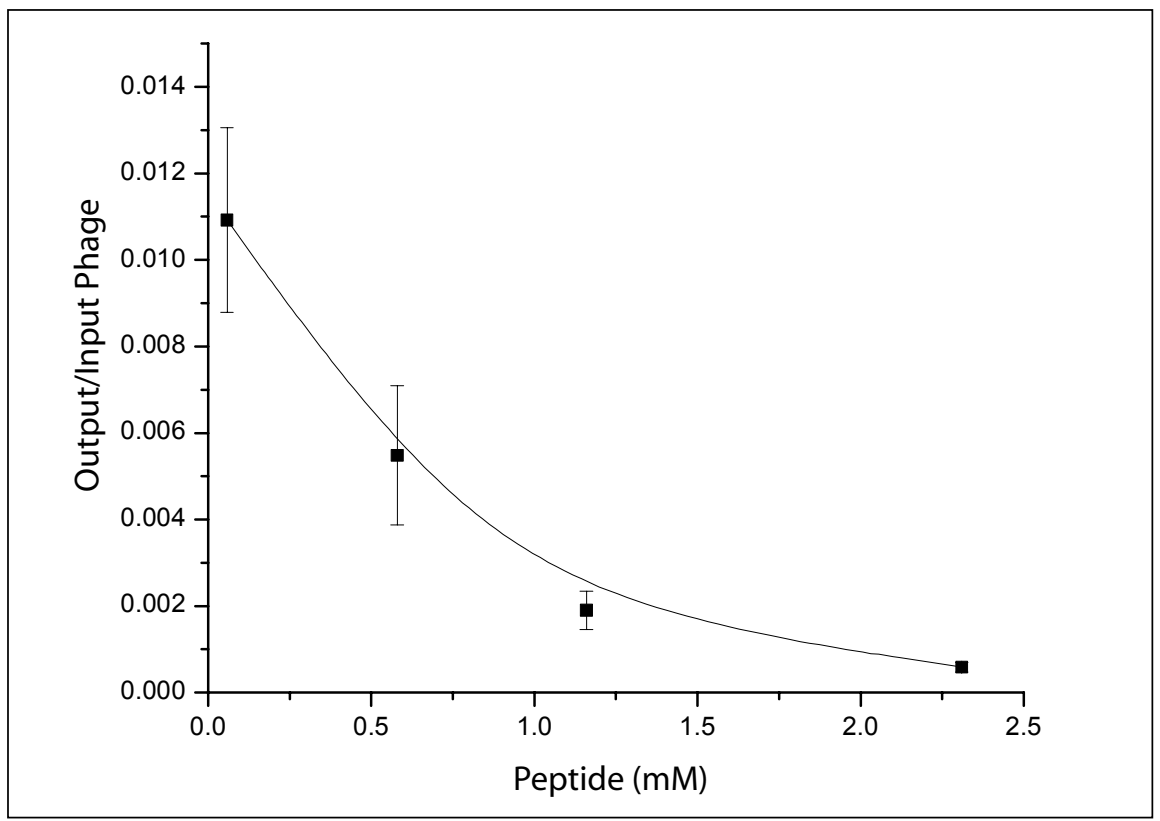

Figure 2. Blocking of Gli27 phage binding to RG2 cells with synthetic RGDS peptide. RG2 cells were incubated with different concentrations of the RGDS peptide followed by Gli27 phage. Phage bound to the cells was quantified via titering and is presented here as an output to input ratio versus peptide concentration. Data represent the means of at least three independent experiments $( \pm \mathrm{SD})$. 
rins. To test whether Gli27 phage-glioma cell recognition occurs via an RGD sequence, we performed competitive assays with a synthetic RGDS peptide. In these experiments, an RGDS peptide was added to the cells prior to the application of the phage. Figure 2 shows that the peptide reduced the phage binding to the cells in a dose-dependent manner. At the lowest concentration of peptide $(0.058 \mathrm{mM})$, phage binding was reduced by $25 \%$. At the highest peptide concentration $(2.31 \mathrm{mM})$, phage binding was inhibited almost entirely.

\section{Phage-Derived Affinity Matrix}

The landscape phage display library used for the identification of the Gli27 clone is based on filamentous phage that belong to $\mathrm{Ff}$ family (fd strain). In this library, the protein coat of each phage particle comprises thousands of identical $\mathrm{pVIII}$ subunits that form a tu-

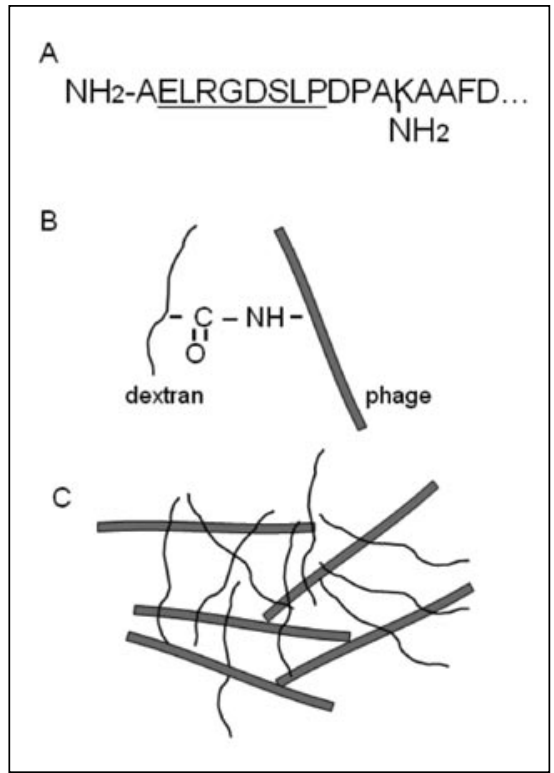

Figure 3. Representation of phage affinity matrix. (A) The first 17 of 55 amino acids of the major coat protein (pVIII) of Gli27 phage are shown. They contain two amino groups (located on the $\mathrm{N}$ terminus and on lysine in the 13th position) that are exposed on the surface of the phage particles and can be modified without compromising phage integrity. (B) The abundance of amino groups on the phage surface (2 in each of 4000 copies of pVIII) allows cross-linkage of phage into an aggregate (matrix) large enough to be pelleted by low-speed centrifugation. (C) In the matrix, phage particles are bound by multiple amide bonds that are formed in the course of the reaction between amino groups of phage proteins and carboxyl groups of dextran polymer. bular sheath surrounding the viral DNA, with the foreign peptide displayed on every subunit (4000 copies total). As shown by Smith et al. (10), such phage particles can be cross-linked to form a hydrophilic matrix similar to those traditionally made from such polymers as cellulose or agarose for affinity chromatography. The matrix is stable at near-neutral $\mathrm{pHs}$ and withstands acidic buffers that are often applied as eluents in affinity purification protocols. Additionally, cross-linked phage (thousands of phage particles linked together) is easily pelleted by low-speed centrifugation, while single phage particles remain in the supernatant.

Using the standard procedure for the preparation of phage described here (15), we obtained approximately $2 \times 10^{14}$ virions that displayed the ELRGDSLP peptide. The whole phage preparation was reacted overnight with the cross-linking agent NHS-dextran. In the course of the reaction, covalent amide bonds were formed between amino groups of phage proteins and carboxyl groups of dextran (Figure 3). The matrix was pelletable by low-speed centrifugation $(12,000 \times g)$ and did not dissociate to monomeric phage particles at a neutral or acidic $\mathrm{pH}$ (data not shown).

\section{Isolation and Identification of Glioma Cell Membrane Proteins}

The isolation of the cell membrane receptor recognized by the Gli27 clone was achieved via affinity precipitation with the cross-linked phage. To form the phage-receptor complexes, cross-linked Gli27 phage was incubated overnight with the clarified lysate of RG2 cells. Following incubation, the complexes were pelleted by centrifugation, and adsorbed proteins were eluted from the phage matrix with a low $\mathrm{pH}$ buffer. Thus, due to multiple copies of glioma-binding peptides expressed on each phage particle, the phage acted as an affinity matrix for the isolation of specific proteins from a mixture of cell proteins. Proteins eluated from cross-linked Gli27 phage were separated by SDS-PAGE, resulting in three bands (approximately 80,
97, and $116 \mathrm{kDa}$ ) visualized by silver staining (data not shown). To show the presence of these proteins on intact RG2 cells, an additional experiment was conducted. Prior to cell lysis and the isolation of the proteins with the phage, intact RG2 cells were biotinylated. Following SDS-PAGE separation, the bands were probed with HRP-labeled streptavidin and visualized with an enhanced chemiluminescence kit (ECL Plus Western Blotting Detection Reagents) (Figure 4A). The figure demonstrates two of the

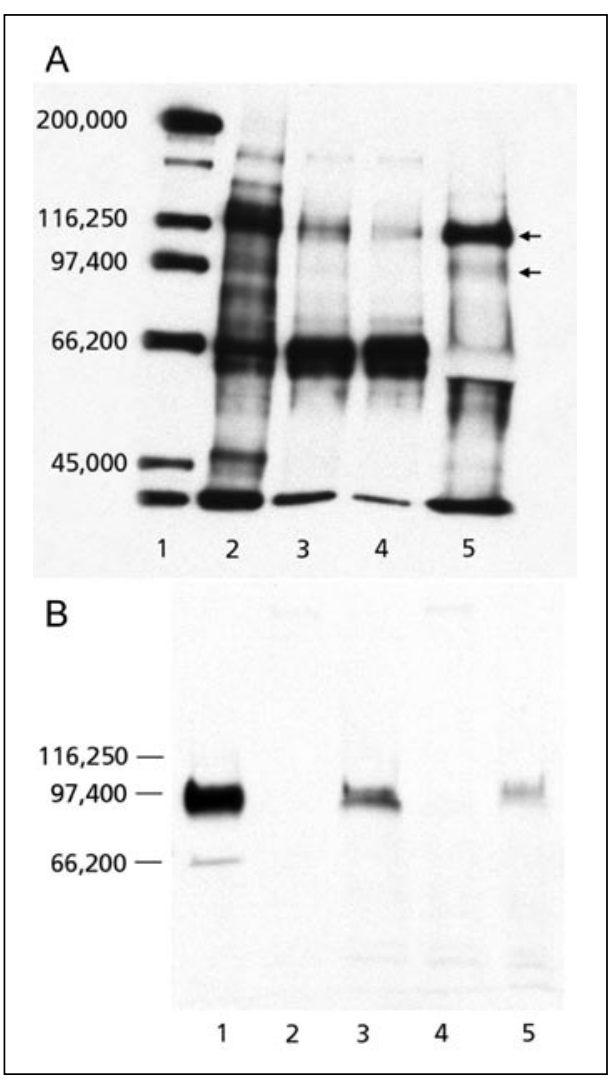

Figure 4. Identification of phage-isolated proteins (A) Western blot of biotinylated RG2 glioma cell-membrane proteins. The proteins were isolated as described in Materials and Methods except that intact RG2 cells were biotinylated prior to cell lysis. Lane 1 contains biotinylated molecular markers (Bio-Rad Laboratories, Hercules, CA, USA). Lane 2 represents cell lysate containing biotinylated cell membrane proteins. Lane 3 is clarified cell lysate (the lane 2 sample after centrifugation to remove insoluble matter). Lane 4 contains cell membrane proteins remaining after Gli27 protein isolation. Lane 5 contains Gli27 phage-isolated protein. (B) Western blot showing the detection of CD44s in Gli27 phage-purified protein preparation and in whole cell lysates. Lanes were loaded with equal levels of total protein and probed with anti-CD44s antibodies (OX49). Lane 1 contains phage-purified protein preparation. Lanes 2 through 5 contain rat whole cell lysates (lane 2, hepatocytes; lane 3, F-98 glioma; lane 4, astrocytes; lane 5, RG2 glioma). 
three bands (approximately 97 and 116 $\mathrm{kDa}$ ) seen with silver staining. The lack of the $80-\mathrm{kDa}$ band could be due to the difference in biotinylation levels of the isolated proteins.

Because the phage clone that was used for affinity purification of the proteins contains the RGD motif, we hypothesized that the receptor for the Gli27 phage might be an integrin with an RGD-binding site. Therefore, antibodies against several RGD-binding receptors, including $\alpha \mathrm{v}, \beta 3$, and $\beta 1$ integrins, which have been shown to be expressed on glioma cells $(16,17)$, were used as probes for protein identification. While the reaction with anti-integrin antibodies did not reveal any positive staining in the phage-purified protein preparation, the reaction with two kinds of anti-CD44 antibodies (one recognizes all CD44 isoforms and the other only standard CD44s) detected one pronounced band of molecular weight approximately $97 \mathrm{kDa}$. A Western blot with OX-49 antibodies that specifically recognize CD44s is shown in Figure 4B. As seen in this figure, CD44s protein is present in whole cell lysates of RG2 and F98 glioma cells (moderate staining) and in the sample of phage-purified protein from RG2 cells (intense staining). A very weak band was observed in normal astrocytes (seen on the film, but not on the film scan shown in Figure 4). No staining was seen in the sample of hepatocytes (negative control).

\section{DISCUSSION}

Recently, the selection of gliomabinding peptide motifs from a landscape phage display library resulted in the identification of Gli27 phage clone (9) that contains an RGD sequence known to recognize a number of integrins. In the present study, Gli27 phage particles were utilized to affinity isolate specific receptors on rat glioma RG2 cells to which they bind.

Gli27 phage was shown to be specific for glioma cells in comparison to four control phage clones that demonstrated background binding to these cells. Phage clones used as controls were identified in unrelated studies $(11,12)$, however, two of them had significant sequence similarities with the phage clone Gli27 selected for glioma. Amino acid compositions of peptides displayed by 1F20 and 1F29 phage clones, which recognize two different binding sites on fibrinogen, are $62.5 \%$ and $75 \%$ identical, respectively, to that of the Gli27 clone. Moreover, 1F20 and Gli27 sequences contain three amino acids in the same position (i.e., glutamic acid in position one, serine in position six, and proline in position eight). Positions two and seven in both of these sequences are occupied by amino acids with nonpolar side chains (two alanines in 1F20 and two leucines in Gli27). Thus, 1F20 and Gli27 differ from each other only by a binding motif, GPR in 1F20 and RGD in Gli27. However, in spite of all similarities, the binding of $1 F 20$ and $1 F 29$ to RG2 glioma cells is three orders of magnitude less than that of the Gli27 that was selected for this type of glioma, confirming Gli27 specificity. To prove that the proteins were isolated via RGD-dependent binding, blocking experiments with synthetic RGDS peptide were performed. It appeared that the RGDS peptide inhibits the interaction between Gli27 phage and glioma cells in a dose-dependent manner.

To create an affinity matrix for receptor isolation, we adapted a method that was developed and tested originally to isolate monoclonal antibodies recognizing S54 epitope on bacteriophage T4 (10). First, individual Gli27 phage particles were linked together by a dextran polymer and then incubated with RG2 cell lysates to allow phage particles to bind to phage-specific proteins. The proteins were eluted from the phage affinity matrix and separated by SDSPAGE. Three polypeptide bands of approximately 80,97 , and $116 \mathrm{kDa}$ were detected on the gel by silver staining. Currently, the only proteins known to bind RGD sequences are integrins. They are heterodimeric transmembrane glycoproteins, consisting of an $\alpha$ and a $\beta$ subunit. Integrins with RGD-binding sites include all $\alpha \mathrm{v}, \alpha \operatorname{IIb} \beta 3, \alpha 5 \beta 1, \alpha 8 \beta 1$, and a few others [paired with a $\beta 1$ subunit, with binding that is weak or only seen under special conditions $(18,19)]$. However, based on our immunoblotting experiments, possible pairing combinations of integrins, and their reported molecular weights $(16,20)$, all of these inte- 
grins can be ruled out as candidates for the isolated proteins. Therefore, the cell surface protein isolated using phage-derived affinity matrix could be an integrin with previously undescribed RGD-binding abilities, an integrin with RGD-binding sites that becomes activated by the presence of phage with RGD peptides, or a novel (non-integrin) protein.

Based on two criteria, the molecular weight (97 kDa) and overexpression in gliomas, a receptor for hyaluronate, CD44, was considered as a candidate for the third polypeptide purified with phage affinity matrix. CD44 is a promiscuous receptor that allows glioma cells to interact with extracellular matrix proteins. The presence of this receptor in human brain tumors has been previously described $(21,22)$. Isolated protein was probed with two types of anti-CD44 antibodies. Both antibodies detected a single band of $97 \mathrm{kDa}$ that corresponds to the standard form of CD44. Because CD44 has no recognized RGD-binding site, it could have been isolated as a part of a complex with another protein, likely an integrin. The presence of CD44 on RG 2 cells was confirmed by immunocytochemistry (data not shown).

In summary, using glioma cell-binding phage (a single clone containing the RGD motif) as an affinity matrix, we isolated not one (as was expected), but three major proteins that are likely to form a complex within the cell membrane. The affinity-isolated material could also contain additional minor polypeptides, both cell membrane and cytoplasmic, which were present at low concentrations and therefore undetectable by silver staining. The complex is composed of RGDbinding protein (most likely two integrin subunits) and CD44s. While the amount of protein obtained by precipitation with $10^{14}$ phage particles was sufficient for immuno-identification via Western blot analysis, it was not adequate for protein sequencing. To overcome this problem, scaled-up methods using at least 10-fold more phage and cell material should be developed. Other methods that might be useful in the identification of phage-isolated proteins include the screening of target cell cDNA libraries against phage display-selected peptides $(2,23)$ or peptide mass spectrometry (3). Such methods might become valuable tools for the identification of novel cell specific recep- tors via their ligands, peptides expressed by phage. The cell specific peptide, the phage bearing the peptide, and the cell receptor characterized with our technique each could be further exploited for diagnostic, prognostic, and therapeutic procedures for malignant gliomas.

\section{ACKNOWLEDGMENTS}

This work was supported by the Scott-Ritchey Research Center, College of Veterinary Medicine, Auburn University (Auburn, AL, USA).

\section{COMPETING INTERESTS STATEMENT}

The authors declare that they have no competing interests.

\section{REFERENCES}

1.Pereira, S., H. Maruyama, D. Siegel, P. Van Belle, D. Elder, P. Curtis, and D. Herlyn. 1997. A model system for detection and isolation of a tumor cell surface antigen using antibody phage display. J. Immunol. Methods 203:11-24.

2.Essler, M. and E. Ruoslahti. 2002. Molecular specialization of breast vasculature: a breasthoming phage-displayed peptide binds to aminopeptidase $P$ in breast vasculature. Proc. Natl. Acad. Sci. USA 99:2252-2257.

3.Gao, C., S. Mao, F. Ronca, S. Zhuang, V. Quaranta, P. Wirsching, and K.D. Janda. 2003. De novo identification of tumor-specific internalizing human antibody-receptor pairs by phage-display methods. J. Immunol. Methods 274:185-197.

4.Barry, M.A., W.J. Dower, and S.A. Johnston. 1996. Toward cell-targeting gene therapy vectors: selection of cell-binding peptides from random peptide-presenting phage libraries. Nat. Med. 2:299-305.

5.Kuan, C.T., C.J. Wikstrand, and D.D. Bigner. 2000. EGFRvIII as a promising target for antibody-based brain tumor therapy. Brain Tumor Pathol. 17:71-78.

6.Mintz, A., D.M. Gibo, B. Slagle-Webb, N.D. Christensen, and W. Debinski. 2002. IL-13R alpha2 is a glioma-restricted receptor for interleukin-13. Neoplasia 4:388-399.

7.Samoylova, T.I., N.E. Morrison, and N.R. Cox. 2003. Molecular markers of glial tumors: current targeting strategies. Curr. Med. Chem. 10:831-843.

8.Petrenko, V.A., G.P. Smith, X. Gong, and T. Quinn. 1996. A library of organic landscapes on filamentous phage. Protein Eng. 9:797-801.

9.Samoylova, T.I., V.A. Petrenko, N. Morrison, L. Globa, H. Baker, and N. Cox. 2003. Phage probes for malignant glioma cells. Mol. Cancer Ther. 2:1-9.
10.Smith, G.P., V.A. Petrenko, and L.J. Matthews. 1998. Cross-linked filamentous phage as an affinity matrix. J. Immunol. Methods 215:151-161

11.Petrenko, V.A., G.P. Smith, M.M. Mazooji, and T. Quinn. 2002. Alpha-helically constrained phage display library. Protein Eng. 15:943-950.

12.Petrenko, V.A. and G.P. Smith. 2000. Phages from landscape libraries as substitute antibodies. Protein Eng. 13:589-592.

13.Barbas III, C.F., D.R. Burton, J.K. Scott, and G.J. Silverman. 2001. Phage Display: A Laboratory Manual. CSH Laboratory Press, Cold Spring Harbor, NY.

14.Ivanenkov, V., F. Felici, and A.G. Menon. 1999. Uptake and intracellular fate of phage display vectors in mammalian cells. Biochim. Biophys. Acta 1448:450-462.

15.Bonnycastle, L.L.C., A. Menendez, and J.K. Scott. 2001. General phage methods, p. 15.115.30. In C.F. Barbas III, D.R. Burton, J.K. Scott, and G.J. Silverman (Eds.), Phage Display: A Laboratory Manual. CSH Laboratory Press, Cold Spring Harbor, NY.

16.Uhm, J.H., C.L. Gladson, and J.S. Rao. 1999 The role of integrins in the malignant phenotype of gliomas. Front Biosci. 4:D188-D199.

17.Rooprai, H.K., T. Vanmeter, C. Panou, S. Schnull, G. Trillo-Pazos, D. Davies, and G.J. Pilkington. 1999. The role of integrin receptors in aspects of glioma invasion in vitro. Int. J. Dev. Neurosci. 17:613-623.

18.Ruoslahti, E. 1996. RGD and other recognition sequences for integrins. Annu. Rev. Cell Dev. Biol. 12:697-715.

19.Schaffner, P. and M.M. Dard. 2003. Structure and function of RGD peptides involved in bone biology. Cell. Mol. Life Sci. 60:119-132.

20.Milner, R., X. Huang, J. Wu, S. Nishimura, R. Pytela, D. Sheppard, and C. ffrench-Constant. 1999. Distinct roles for astrocyte $\alpha v \beta 5$ and $\alpha v \beta 8$ integrins in adhesion and migration. J. Cell Sci. 112:4271-4279

21.Ranuncolo, S.M., V. Ladeda, S. Specterman, M. Varela, J. Lastiri, A. Morandi, E. Matos, E. Bal De Kier Joffe, et al. 2002. CD44 expression in human gliomas. J. Surg. Oncol. 79:30-36.

22.Akiyama, Y., S. Jung, B. Salhia, S. Lee, S. Hubbard, M. Taylor, T. Mainprize, K. Akaishi, et al. 2001. Hyaluronate receptors mediating glioma cell migration and proliferation. J. Neurooncol. 53:115-127.

23.Li, J., S. Pereira, P. Van Belle, P. Tsui, D. Elder, D. Speicher, K. Deen, A. Linnenbach, et al. 2001. Isolation of the melanoma-associated antigen p23 using antibody phage display. J. Immunol. 166:432-438.

Received 20 February 2004; accepted 23 April 2004.

\section{Address correspondence to:}

Tatiana I. Samoylova

The Scott-Ritchey Research Center

College of Veterinary Medicine

Auburn University

Auburn, AL 36849, USA

e-mail:samoiti@vetmed.auburn.edu 